\title{
Breakdancing on actin
}

ARP2/3-

mediated actin polymerization

chromatin

dynamics

during HDR promotes

The nucleus contains monomeric actin, which can interact with various chromatin regulators and modulate their activity. Notably, actin polymerization into filamentous F-actin can also occur in certain conditions, including genotoxic stress. Nevertheless, the role of F-actin in the nucleus remains largely unexplored. Two studies published in Nature now show the importance of nuclear actin polymerization for chromatin dynamics during repair of DNA double-strand breaks (DSBs) by homology-directed repair (HDR).

By performing an unbiased proteomics screen of DSB-containing chromatin-protein complexes from frog egg extracts, Schrank et al. established that DSBs can associate with actin and its regulators. Among these was the actin-related protein $2 / 3$ (ARP2/3) complex, which promotes the formation of branched actin networks. Co-localization of ARP2/3 with DSBs was further demonstrated in mammalian cell lines treated with a DSB-inducing antibiotic, neocarzinostatin (NCS). Notably, this

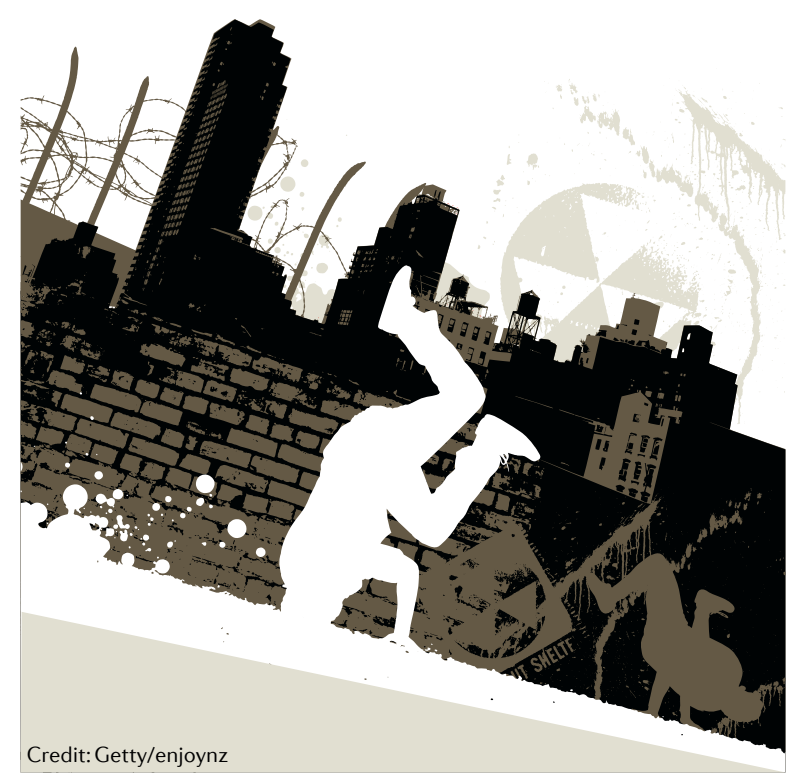

co-localization was dependent on DSB signalling and was specific to DSBs targeted for repair via HDR (bound by RAD51). NCS treatment also promoted the formation of nuclear actin foci at RAD51containing DSBs. Thus, branched actin networks are polymerized around DSBs during HDR.

Chromatin is highly dynamic during HDR, which involves considerable motion of chromatin regions and clustering of DSBs into distinct foci. Chromatin motion, the frequency of DSB clustering and the size of DSB clusters were all reduced following ARP2/3 inhibition. Furthermore, inhibition of ARP2/3, its activator WASP (Wiskott-Aldrich syndrome protein) or interference with nuclear actin polymerization lowered the efficiency of DSB repair, specifically through homologous recombination. Thus, ARP2/3-mediated actin polymerization promotes chromatin dynamics during HDR, thereby supporting DSB repair.

DNA repair, especially through HDR, is particularly challenging in heterochromatin owing to the presence of repetitive sequences. Notably, HDR in heterochromatin is associated with relocalization of heterochromatic DSBs to the nuclear periphery, which has been proposed to prevent aberrant recombination between non-homologous, repetitive regions.

Caridi, D’Agostino et al. showed that in irradiated fly and mouse cells heterochromatic DSB relocalization depends on actin polymerization and ARP2/3. In line with this, in irradiated fly cells, actin polymerized around the heterochromatin 'domain' and formed Arp2/3-enriched filaments that departed from repair sites and were directed towards the nuclear periphery. Formation of these filaments was required for relocalization of heterochromatic DSBs, and DSB foci bound by HDR machinery were found to travel in a directed fashion along these filaments. This suggests that actin filaments serve as tracks for directional transport of heterochromatic DSBs during HDR. This transport seems to be driven by myosins, as knockdown of myosin I or myosin $\mathrm{V}$ (which were previously shown to function in the context of chromatin) or of myosin chaperone Unc45 (a myosin activator) caused defects in heterochromatic DSB relocalization.

Interference with nuclear actin polymerization or depletion of Arp2/3, myosins or Unc45 all hampered repair of heterochromatic DSBs in irradiated fly cells and decreased cell survival and genome stability. Moreover, inactivation of myosins or interference with actin polymerization led to relocalization defects and genomic instability in mouse cells. Thus, transport of heterochromatic DSBs on actin filaments is a conserved mechanism that supports HDR and the maintenance of genomic integrity.

In summary, by enabling chromatin dynamics, nuclear actin networks are important for efficient, error-free HDR. In the future, it would be interesting to study how actin polymerization and actin-dependent chromatin dynamics are regulated in the context of DNA damage repair.

Paulina Strzyz

ORIGINAL ARTICLES Schrank, B. R. et al. Nuclear ARP2/3 drives DNA break clustering for homology-directed repair. Nature https://doi.org/ 10.1038/s41586-018-0237-5 (2018)|Caridi, C. P., D'Agostino, C. et al. Nuclear F-actin and myosins drive relocalization of heterochromatic breaks. Nature https://doi.org/10.1038/s41586-0180242-8 (2018)

FURTHER READING Grosse, R. \& Vartiainen M. K. To be or not to be assembled: progressing into nuclear actin filaments. Nat. Rev. Mol. Cell Biol. 14, 693-697 (2013) 